\title{
Other High-Risk Conditions
}

\section{Executive Summary of the Japan Atherosclerosis Society (JAS) Guidelines for the Diagnosis and Prevention of Atherosclerotic Cardiovascular Diseases in Japan - 2012 Version}

\author{
Tamio Teramoto, Jun Sasaki, Shun Ishibashi, Sadatoshi Birou, Hiroyuki Daida, Seitaro Dohi, Genshi Egusa, \\ Takafumi Hiro, Kazuhiko Hirobe, Mami lida, Shinji Kihara, Makoto Kinoshita, Chizuko Maruyama, \\ Takao Ohta, Tomonori Okamura, Shizuya Yamashita, Masayuki Yokode and Koutaro Yokote
}

Committee for Epidemiology and Clinical Management of Atherosclerosis

\section{History of Coronary Artery Disease (CAD)}

Epidemiological studies and interventional trials conducted in Western countries and the results of a meta-analysis of these studies have revealed that the incidence of cardiovascular events in patients with a history of CAD is higher than that observed in primary prevention patients.

In Japan, the incidence of cardiovascular events in primary prevention subjects in the J-LIT trial was $0.9 / 1,000$ person-years ${ }^{1)}$, while that in secondary prevention patients was higher, with a value of 4.5/1,000 person-years ${ }^{2}$. The $\mathrm{JCAD}^{3)}$ and CREDO-Kyoto ${ }^{4)}$, registration studies of patients with $\mathrm{CAD}$, reported the incidence of cardiovascular events to be $\geq 15 / 1,000$ person-years. Among secondary prevention patients, there are further high-risk conditions, including acute coronary syndrome, smoking, diabetes mellitus, metabolic syndrome, chronic kidney disease, noncardiogenic cerebral infarction, peripheral artery disease and a constellation of risk factors. It has been reported that these patients clearly have a high incidence of recurrent coronary events, even when the LDL cholesterol (LDL-C) level is managed to the same extent as that in patients without complications.

\section{Cerebrovascular Disease}

It is well known that patients with a history of cerebrovascular disease are at a high risk for CAD.

It has been reported that the annual incidence of myocardial infarction in stroke patients ranges from $0.40 \%$ to $0.45 \%$ ( 4.0 to 4.5 persons $/ 1,000$ personyears) in Japan ${ }^{5,6)}$. Based on these figures, the incidence of myocardial infarction over 10 years in stroke

Received: December 6, 2012

Accepted for publication: June 3, 2013 patients is approximately $3.9 \%$ to $4.4 \%$, suggesting that Japanese stroke patients are also at a high risk of developing $\mathrm{CAD}$. In particular, noncardiogenic cerebral infarctions are derived from atherosclerotic lesions and are therefore a high-risk condition for CAD.

\section{Chronic Kidney Disease (CKD)}

Chronic kidney disease (CKD) is defined as the presence of kidney damage and/or a decreased kidney function lasting for $\geq 3$ months. The former is determined according to the levels of albuminuria/proteinuria, and the latter is evaluated based on a decreased glomerular filtration rate (GFR). In the Evidencebased Practice Guidelines for the Treatment of CKD 2009 issued by the Japanese Society of Nephrology, CKD is divided into stages 1 to 5, with a therapeutic plan proposed for each stage ${ }^{7)}$ (see footnote).

$\mathrm{CKD}$ is a high-risk condition for cardiovascular disease $(\mathrm{CVD})^{8)}$. Large-scale observational cohort studies conducted in the Japanese general population, including the NIPPON DATA80, Suita study and JALS-ECC trials ${ }^{9-13)}$, have demonstrated that CKD is associated with an approximately 2-fold higher risk of CVD. In a post hoc analysis of the CASE-J trial investigating the effects of antihypertensive agents on CVD in Japanese patients with hypertension ${ }^{14)}$, the relative risks associated with various risk factors were compared. The analysis showed that CKD exhibits a significant association with cardiovascular risks (relative risk: 2.8) that is comparable to or even stronger than that with a history of cerebrovascular disease (relative risk: 2.2), heart disease (relative risk: 2.2) or type 2 diabetes (relative risk: 2.0).

The exacerbation of classical risk factors associated with CKD, such as blood pressure, the lipid levels and glucose metabolism, contributes to the 
increased risk of CVD observed in patients with $\mathrm{CKD}^{8)}$. Regarding lipids, $\mathrm{CKD}$ is a representative cause of secondary hyperlipidemia; nephrotic syndrome ${ }^{15)}$ is often accompanied by hyper-LDL cholesterolemia, while chronic renal failure ${ }^{16)}$ is often accompanied by hypertriglyceridemia due to the accumulation of remnant lipoproteins or a high VLDL level and hypo-HDL cholesterolemia. The non HDL cholesterol (HDL-C) level, the sum of the levels of cholesterol in TG-rich lipoproteins and LDL, has been reported to be an independent factor associated with the carotid artery intima-media thickness (IMT) ${ }^{17)}$ and pulse wave velocity (PWV) ${ }^{18)}$ in CKD patients. The ARIC ${ }^{19)}$, an epidemiological study conducted in the US general population, demonstrated that higher total cholesterol (TC) or triglycerides (TG) levels are associated with a higher risk of incident $\mathrm{CAD}$, regardless of the GFR. In contrast, the HDL-C level is not associated with a risk of CAD in patients with a low GFR, suggesting that the non HDL-C level is related to the development of CAD in CKD patients with a low GFR. Therefore, dyslipidemia is closely associated with CVD in patients with CKD.

The risk of CVD is high in the presence of CKD. There is, however, controversy over whether CKD itself is the cause of CVD. It may be that there are common risk factors that adversely affect both CKD and CVD. Meanwhile, in a model adjusted for classical risk factors, the presence or absence of CKD was found to be independently associated with CVD, suggesting the involvement of non-classical risk factors associated with $\mathrm{CKD}^{8)}$. In any case, $\mathrm{CKD}$ should be treated as a high-risk condition for $\mathrm{CVD}^{20)}$.

\section{Footnote}

$\mathrm{CKD}$ is currently classified based on the cause, GFR category and albuminuria (or proteinuria) category (CGA), as proposed by the 'KDIGO 2012 Clinical Practice Guidelines for the Evaluation and Management of Chronic Kidney Disease' and the 'Clinical Practice Guidebook for the Diagnosis and Treatment of Chronic Kidney Disease 2012' issued by the Japanese Society of Nephrology.

\section{Peripheral Arterial Disease (PAD) and Abdominal Aortic Aneurysm (AAA)}

Peripheral arterial disease was traditionally called ASO (arteriosclerosis obliterans) in Japan; however, in these guidelines, the term PAD ${ }^{21)}$ is used and the disease is defined as the presence of stenotic/obstructive lesions caused by atherosclerosis of the arteries in the extremities. PAD is characterized by symptoms such as coldness of the lower extremities, intermittent claudication, ulcers and necrosis. Abdominal aortic aneurysm (AAA) is a condition involving plaque formation and ulceration in the luminal face, as well as external saccular aneurysm formation due to atherosclerosis of the abdominal aorta. These atherosclerotic diseases (including carotid artery stenosis and renal artery stenosis) require treatments such as revascularization; however, managing the causative risk factors is also important. It should also be noted that the primary cause of death in patients with these diseases is CAD or cerebrovascular disease. Although epidemiological studies conducted in Western countries revealed long ago that these diseases are high-risk conditions for CVD, this has only recently been reported in Japan.

The REACH registry, a prospective epidemiological study, reported that the incidence of CVD per year in 603 patients with coexisting PAD among 5,193 Japanese patients entered until 2004 was $1.25 \%$ for all deaths, $0.55 \%$ for cardiovascular death, $0.77 \%$ for nonfatal myocardial infarction, $1.56 \%$ for nonfatal stroke, $3.08 \%$ for cardiovascular death + nonfatal myocardial infarction + nonfatal stroke and 10.52\% for cardiovascular death + nonfatal myocardial infarction + nonfatal stroke + hospitalization ${ }^{5)}$. These values are comparable to those for patients with coexisting CAD. Furthermore, in a prospective observational study of 557 patients with PAD, Shigematsu et al. reported that the incidence of CVD over three years was $6.3 \%$ for cardiovascular death, $11.3 \%$ for heart disease, $7.0 \%$ for brain disease and $16.9 \%$ for lower extremity events ${ }^{22)}$.

Regarding AAA, Kioka et al. performed preoperative coronary angiography in 94 Japanese patients who underwent elective surgery for AAA (81 men; mean age: $71.7 \pm 6.4$ years) and reported that complications of CAD were observed in $45.7 \%$ of the patients ${ }^{23)}$. Similarly, Takigawa et al. reported that the complication rate for asymptomatic CAD detected on coronary angiography in 201 Japanese patients who underwent elective surgery for AAA (161 men; mean: age $73.1 \pm 7.7$ years) was $29.4 \%{ }^{24)}$. Hirose et al. performed ATP-loading myocardial single-photon emission computed tomography (SPECT) in a total of 788 Japanese patients, including 500 patients with aortic aneurysms, 183 patients with lower extremity PAD and 105 patients with combined aortic aneurysms and lower extremity PAD, who had not been diagnosed with $\mathrm{CAD}$ and reported that myocardial ischemia was observed in $77 \%$ of the patients with combined aortic aneurysms and PAD, 55\% of the patients with PAD and $37 \%$ of the patients with aortic aneurysms ${ }^{25}$. These reports demonstrate that the existence of PAD 
and AAA is also an important high-risk condition for CVD in Japanese patients.

Atherosclerotic findings in the carotid artery are independent risk factors for $\mathrm{CVD}^{26,27)}$. Thickening of the common carotid artery IMT on ultrasonography is associated with an increased incidence of CVD. In addition, the existence of plaque and its characteristics (e.g., low-intensity features and the formation of ulcers) is involved in the development of $\mathrm{CVD}^{28,29)}$. In Japan, it has been reported that IMT is an independent significant predictive factor for $\mathrm{CAD}$ as well as cerebral infarction ${ }^{30-32)}$. In several reports, the extent of IMT has been found to be significantly correlated with the extent of coronary atherosclerosis ${ }^{33}$ 34). Shimada et al. reported that $37 \%$ of patients with carotid artery stenosis who underwent carotid endarterectomy presented with coronary lesions on preoperative coronary angiography, half of whom presented with triplevessel disease or left main $\mathrm{CAD}^{35)}$.

Atherosclerotic renal artery stenosis is a cause of renovascular hypertension. It has been reported that $5 \%$ to $22 \%$ of elderly patients with CKD have coexisting renal artery stenosis. Renal artery stenosis is also a risk factor for CVD, and the complication rate for atherosclerotic disease in other organs is high ${ }^{7,36-38)}$. In the primary prevention of CVD, carotid artery findings and renal artery stenosis should be noted.

As noted above, it has been reported that AAA, carotid artery lesions and renal artery stenosis are associated with CVD; however, it is difficult to say that sufficient results of prospective studies in Japan have been accumulated. Therefore, these guidelines include only PAD as a high-risk condition.

\section{Footnotes}

This is an English version of the guidelines of the Japan Atherosclerosis Society (Chapter 6) published in Japanese in June 2012.

\section{Acknowledgements}

We are grateful to the following societies for their collaboration and valuable contributions: Dr. Hidenori Arai (The Japan Geriatrics Society), Dr. Kiminori Hosoda (Japan Society for the Study of Obesity), Dr. Hiroyasu Iso (Japan Epidemiological Association), Dr. Atsunori Kashiwagi (Japan Diabetes Society), Dr. Masayasu Matsumoto (The Japan Stroke Society), Dr. Hiromi Rakugi (The Japanese Society of Hypertension), Dr. Tetsuo Shoji (Japanese Society of Nephrology) and Dr. Hiroaki Tanaka (Japanese Society of Physical Fitness and Sports Medicine). We also thank
Dr. Shinji Koba, Dr. Manabu Minami, Dr. Tetsuro Miyazaki, Dr. Hirotoshi Ohmura, Dr. Mariko HaradaShiba, Dr. Hideaki Shima, Dr. Daisuke Sugiyama, Dr. Minoru Takemoto and Dr. Kazuhisa Tsukamoto for supporting this work.

\section{References}

1) Matsuzaki M, Kita T, Mabuchi H, Matsuzawa Y, Nakaya N, Oikawa S, Saito Y, Sasaki J, Shimamoto K, Itakura H: J-LIT Study Group: Large scale cohort study of the relationship between serum cholesterol concentration and coronary events with low-dose simvastatin therapy in Japanese patients with hypercholesterolemia: primary prevention cohort study of the Japan Lipid Intervention Trial (J-LIT). Circ J, 2002; 66: 1087-1095

2) Mabuchi H, Kita T, Matsuzaki M, Matsuzawa $Y$, Nakaya N, Oikawa S, Saito Y, Sasaki J, Shimamoto K, Itakura H; J-LIT Study Group. Japan Lipid Intervention Trial: Large scale cohort study of the relationship between serum cholesterol concentration and coronary events with low-dose simvastatin therapy in Japanese patients with hypercholesterolemia and coronary heart disease: secondary prevention cohort study of the Japan Lipid Intervention Trial (J-LIT). Circ J, 2002; 66: 1096-1100

3) Japanese Coronary Artery Disease (JCAD) Study Investigators: Current status of the background of patients with coronary artery disease in Japan. Circ J, 2006; 70: 12561262

4) Furukawa $Y$, Taniguchi R, Ehara N, Ozasa N, Haruna $Y$, Saito N, Doi T, Hoshino K, Shizuta S, Morimoto T, Imai Y, Teramukai S, Fukushima M, Kita T, Kimura T; CREDO-Kyoto Investigators: Better survival with statin administration after revascularization therapy in Japanese patients with coronary artery disease: perspectives from the CREDO-Kyoto registry. Circ J, 2008; 72: 1937-1945

5) Uchiyama $S$, Goto $S$, Matsumoto $M$, Nagai R, Origasa $H$, Yamazaki T, Shigematsu H, Shimada K, Yamada N, Bhatt DL, Steg PG, Ikeda Y; REduction of Atherothrombosis for Continued Health Registry Investigators: Cardiovascular event rates in patients with cerebrovascular disease and atherothrombosis at other vascular locations: Results from 1-year outcomes in the Japanese REACH Registry. J Neurol Sci, 2009; 287: 45-51

6) Goto S, Ikeda Y, Shimada K, Uchiyama S, Origasa H, Kobayashi H; The J-TRACE Investigators: One-year cardiovascular event rates in Japanese outpatients with myocardial infarction, stroke, and atrial fibrillation. Circ J, 2011; 75: 2598-2604

7) Japanese Society of Nephrology (editor): Evidence-based practice guideline for the treatment of CKD 2009. Tokyo Igakusha, 2009

8) Sarnak MJ, Levey AS, Schoolwerth AC, Coresh J, Culleton B, Hamm LL, McCullough PA, Kasiske BL, Kelepouris E, Klag MJ, Parfrey P, Pfeffer M, Raij L, Spinosa DJ, Wilson PW ; American Heart Association Councils on Kidney in Cardiovascular Disease, High Blood Pressure Research, Clinical Cardiology, and Epidemiology and Prevention: Kidney disease as a risk factor for develop- 
ment of cardiovascular disease: a statement from the American Heart Association Councils on Kidney in Cardiovascular Disease, High Blood Pressure Research, Clinical Cardiology, and Epidemiology and Prevention. Circulation, 2003; 108: 2154-2169

9) Ninomiya T, Kiyohara Y, Kubo M, Tanizaki Y, Doi Y, Okubo K, Wakugawa Y, Hata J, Oishi Y, Shikata K, Yonemoto K, Hirakata H, Iida M: Chronic kidney disease and cardiovascular disease in general Japanese population: the Hisayama study. Kidney Int, 2005; 68: 228-236

10) Ninomiya T, Kiyohara Y, Tokuda Y, Doi Y, Arima H, Harada A, Ohashi Y, Ueshima H; Japan Arteriosclerosis Longitudinal Study Group: Impact of kidney disease and blood pressure on the development of cardiovascular disease: An overview from the Japan Arteriosclerosis Longitudinal Study. Circulation, 2008; 118: 2694-2701

11) Nakamura K, Okamura T, Hayakawa T, Kadowaki T, Kita Y, Ohnishi H, Saitoh S, Sakata K, Okayama A, Ueshima H; NIPPON DATA90 Research Group: Chronic kidney disease is a risk factor for cardiovascular death in a community-based population in Japan: Nippon Data 90. Circ J, 2006; 70: 954-959

12) Kokubo Y, Nakamura S, Okamura T, Yoshimasa Y, Makino H, Watanabe M, Higashiyama A, Kamide K, Kawanishi K, Okayama A, Kawano Y: Relationship between blood pressure category and incidence of stroke and myocardial infarction in an urban Japanese population with and without chronic kidney disease: the Suita study. Stroke, 2009; 40: 2674-2679

13) Irie F, Iso H, Sairenchi T, Fukasawa N, Yamagishi K, Ikehara S, Kanashiki M, Saito Y, Ota H, Nose T: The relationships of proteinuria, serum creatinine, glomerular filtration rate with cardiovascular disease mortality in Japanese general population. Kidney Int, 2006; 69: 12641271

14) Yasuno S, Ueshima K, Oba K, Fujimoto A, Ogihara T, Saruta T, Nakao K: Clinical significance of left ventricular hypertrophy and changes in left ventricular mass in highrisk hypertensive patients: a subanalysis of the candesartan antihypertensive survival evaluation in Japan trial. J Hypertens, 2009; 27: 1705-1712

15) Kronenberg F: Dyslipidemia and nephrotic syndrome: recent advances. J Ren Nutr, 2005; 15: 195-203

16) Wanner C, Quaschning T: Dyslipidemia and renal disease: pathogenesis and clinical consequences. Curr Opin Nephrol Hypertens, 2001; 10: 195-201

17) Shoji T, Emoto M, Tabata T, Kimoto E, Shinohara K, Maekawa K, Kawagishi T, Tahara H, Ishimura E, Nishizawa Y: Advanced atherosclerosis in predialysis patients with chronic renal failure. Kidney Int, 2002; 61: 2187-2192

18) Shinohara K, Shoji T, Tsujimoto Y, Kimoto E, Tahara H, Koyama H, Emoto M, Ishimura E, Miki T, Tabata T, Nishizawa Y: Arterial stiffness in predialysis patients with uremia. Kidney Int, 2004; 65: 936-943

19) Muntner P, He J, Astor BC, Folsom AR, Coresh J: Traditional and nontraditional risk factors predict coronary heart disease in chronic kidney disease: results from the atherosclerosis risk in communities study. J Am Soc Nephrol, 2005; 16: 529-538
20) Shoji T, Abe T, Matsuo H, Egusa G, Yamasaki Y, Kashihara N, Shirai K, Kashiwagi A; Committee of Renal and Peripheral Arteries, Japan Atherosclerosis Society: Chronic kidney disease, dyslipidemia, and atherosclerosis. J Atheroscler Thromb, 2012; 19: 299-315

21) Hirsch AT, Haskal ZJ, Hertzer NR, Bakal CW, Creager MA, Halperin JL, Hiratzka LF, Murphy WR, Olin JW, Puschett JB, Rosenfield KA, Sacks D, Stanley JC, Taylor LM Jr, White CJ, White J, White RA, Antman EM, Smith SC Jr, Adams CD, Anderson JL, Faxon DP, Fuster V, Gibbons RJ, Hunt SA, Jacobs AK, Nishimura R, Ornato JP, Page RL, Riegel B; American Association for Vascular Surgery; Society for Vascular Surgery; Society for Cardiovascular Angiography and Interventions; Society for Vascular Medicine and Biology; Society of Interventional Radiology; ACC/AHA Task Force on Practice Guidelines Writing Committee to Develop Guidelines for the Management of Patients With Peripheral Arterial Disease; American Association of Cardiovascular and Pulmonary Rehabilitation; National Heart, Lung, and Blood Institute; Society for Vascular Nursing; TransAtlantic Inter-Society Consensus; Vascular Disease Foundation: ACC/AHA 2005 Practice Guidelines for the management of patients with peripheral arterial disease (lower extremity, renal, mesenteric, and abdominal aortic): a collaborative report from the American Association for Vascular Surgery/Society for Vascular Surgery, Society for Cardiovascular Angiography and Interventions, Society for Vascular Medicine and Biology, Society of Interventional Radiology, and the ACC/AHA Task Force on Practice Guidelines (Writing Committee to Develop Guidelines for the Management of Patients With Peripheral Arterial Disease): endorsed by the American Association of Cardiovascular and Pulmonary Rehabilitation; National Heart, Lung, and Blood Institute; Society for Vascular Nursing; TransAtlantic Inter-Society Consensus; and Vascular Disease Foundation. Circulation, 2006; 113: e463e654

22) Shigematsu H, Nishibe T, Obitsu Y, Matsuzaki K, Ishida A, Miyata T, Shindo S, Hida K, Ohta T, Ando M, Kawasaki T, Yasugi T, Matsumoto T: Three year cardiovascular events and disease progress in patients with peripheral arterial disease: results from the Japan Medication Therapy for Peripheral Arterial Disease (J-METHOD). Int Angiol, 2010; 29(Suppl 1-2): 2-13

23) Kioka Y, Tanabe A, Kotani Y, Yamada N, Nakahama M, Ueda T, Seitou T, Maruyama M: Review of coronary artery disease in patients with infrarenal abdominal aortic aneurysm. Circ J, 2002; 66: 1110-1112

24) Takigawa M, Yokoyama N, Yoshimuta T, Takeshita S: Prevalence and prognosis of asymptomatic coronary artery disease in patients with abdominal aortic aneurysm and minor or no perioperative risks. Circ J, 2009; 73: 1203 1209

25) Hirose K, Chikamori T, Hida S, Tanaka H, Igarashi Y, Watanabe Y, Koizumi N, Kawaguchi S, Obitsu Y, Shigematsu H, Yamashina A: Prevalence of coronary heart disease in patients with aortic aneurysm and/or peripheral artery disease. Am J Cardiol, 2009; 103: 1215-1220

26) O’Leary DH, Polak JF, Kronmal RA, Manolio TA, Burke 
GL, Wolfson SK Jr: Carotidartery intima and media thickness as a risk factor for myocardial infarction and stroke in older adults. Cardiovascular Health Study Collaborative Research Group. N Engl J Med, 1999; 340: $14-22$

27) Lorenz MW, Markus HS, Bots ML, Rosvall M, Sitzer M: Prediction of clinical cardiovascular events with carotid intimamedia thickness: a systematic review and metaanalysis. Circulation, 2007; 115: 459-467

28) Mathiesen EB, Bønaa KH, Joakimsen O: Echolucent plaques are associated with high risk of ischemic cerebrovascular events in carotid stenosis: the tromsø study. Circulation, 2001; 103: 2171-2175

29) Nakamura T, Tsutsumi Y, Shimizu Y, Uchiyama S: Ulcerated carotid plaques with ultrasonic echolucency are causatively associated with thromboembolic cerebrovascular events. J Stroke Cerebrovasc Dis. 2011 Aug 4. [Epub ahead of print]

30) Kitagawa K, Hougaku $H$, Yamagami $H$, Hashimoto $H$, Itoh T, Shimizu Y, Takahashi D, Murata S, Seike Y, Kondo K, Hoshi T, Furukado S, Abe Y, Yagita Y, Sakaguchi M, Tagaya M, Etani H, Fukunaga R, Nagai Y, Matsumoto M, Hori M; OSACA2 Study Group: Carotid intima-media thickness and risk of cardiovascular events in highrisk patients. Results of the Osaka Follow-Up Study for Carotid Atherosclerosis 2 (OSACA2 Study). Cerebrovasc Dis, 2007; 24: 35-42

31) Irie $Y$, Katakami N, Kaneto H, Kasami R, Sumitsuji $S$, Yamasaki K, Tachibana K, Kuroda T, Sakamoto K, Umayahara Y, Ueda Y, Kosugi K, Shimomura I: Maximum carotid intima-media thickness improves the prediction ability of coronary artery stenosis in type 2 diabetic patients without history of coronary artery disease. Atherosclerosis, 2012; 221: 438-444 (Epub 2012 Jan 21)

32) Hirano M, Nakamura T, Kitta Y, Takishima I, Deyama J, Kobayashi T, Fujioka D, Saito Y, Watanabe K, Watanabe
Y, Kawabata K, Obata JE, Kugiyama K: Short-term progression of maximum intima-media thickness of carotid plaque is associated with future coronary events in patients with coronary artery disease. Atherosclerosis, 2011; 215: 507-512

33) Teragawa H, Kato M, Kurokawa J, Yamagata T, Matsuura $\mathrm{H}$, Chayama K: Usefulness of flow-mediated dilation of the brachial artery and/or the intima-media thickness of the carotid artery in predicting coronary narrowing in patients suspected of having coronary artery disease. Am J Cardiol, 2001; 88: 1147-1151

34) Kasami R, Kaneto H, Katakami N, Sumitsuji S, Yamasaki K, Kuroda T, Tachibana K, Yasuda T, Kuroda A, Matsuoka TA, Matsuhisa M, Shimomura I: Relationship between carotid intima-media thickness and the presence and extent of coronary stenosis in type 2 diabetes patients with carotid atherosclerosis but without history of coronary artery disease. Diabetes Care, 2011; 34: 468-470

35) Shimada T, Toyoda K, Inoue T, Kamouchi M, Matsumoto T, Hiyamuta K, Imaizumi T, Okada Y: Prediction of coronary artery disease in patients undergoing carotid endarterectomy. J Neurosurg, 2005; 103: 593-596

36) Nakamura $S$, Iihara $K$, Matayoshi $T$, Yasuda $H$, Yoshihara F, Kamide K, Horio T, Miyamoto S, Kawano Y: The incidence and risk factors of renal artery stenosis in patients with severe carotid artery stenosis. Hypertens Res, 2007; 30: 839-844

37) Uzu T, Inoue T, Fujii T, Nakamura $S$, Inenaga T, Yutani C, Kimura G: Prevalence and predictors of renal artery stenosis in patients with myocardial infarction. Am J Kidney Dis, 1997; 29: 733-738

38) Yamashita T, Ito F, Iwakiri N, Mitsuyama H, Fujii S, Kitabatake A: Prevalence and predictors of renal artery stenosis in patients undergoing cardiac catheterization. Hypertens Res, 2002; 25: 553-557 